Artigo / Article

\title{
Fatores prognósticos nas síndromes mielodisplásicas
}

\section{Prognostic factors for myelodysplastic syndromes}

Alexandre G. Apa $^{1}$

Cibelli N. R. M. Gutz ${ }^{2}$
As síndromes mielodisplásicas compreendem um conjunto heterogêneo de doenças hematopoéticas que se caracterizam por hematopoese ineficaz e se apresentam geralmente com citopenias no sangue periférico, medula óssea hipercelular e displasia na diferenciação celular. Vários fatores clínicos e laboratoriais foram analisados como prognósticos. O objetivo dessa revisão é analisar os sistemas prognósticos avaliando sobrevida global e abordagem terapêutica. A avaliação do sistema WPSS, que alia grupos de riscos citogenéticos e a presença ou não de dependência transfusional define cinco grupos de riscos com diferença estatística em termos de sobrevida global e risco de transformação leucêmica. A proposta formulada é a avaliação do sistema WPSS como sistema prognóstico capaz de substituir o IPSS a fim de melhor definir os grupos de risco e diferentes abordagens terapêuticas. Rev. bras. hematol. hemoter. 2006; 28(3):198-200.

Palavras-chave: Prognóstico; síndrome mielodisplásica.

\section{Introdução}

Nas mielodisplasias, vários fatores clínicos e laboratoriais foram avaliados como fatores prognósticos, porém poucos mostraram significância estatística quando avaliados como variável independente numa análise multivariada (citogenética e dependência transfusional). ${ }^{1}$ Os principais fatores prognósticos estudados são: idade, mielodisplasia primária versus secundária, número de blastos medulares, número de citopenias periféricas, número de linhagens hematopoiéticas displásicas, presença de ALIP na biópsia de medula óssea, níveis séricos de LDH e TDK, imunofenotipagem, expressão gênica molecular, expressão gênica (microarray), citogenética, cultura de células, dependência e carga transfusional. ${ }^{1,2,3}$ As mielodisplasias secundárias são as relacionadas ao tratamento prévio com radioterapia e/ou quimioterapia e sua pior evolução está relacionada a alterações citogenéticas de mau prognóstico.

Baseado nesses fatores, várias classificações e sistemas prognósticos foram criados visando definir melhor as síndromes mielodisplásicas quanto à sobrevida global e ris- co de transformação leucêmica. Os principais são: classificação FAB, ${ }^{4}$ classificação WHO, ${ }^{5}$ Escore MDS/ WHO, ${ }^{6}$ escore prognóstico internacional IPSS ${ }^{3}$ e escore WPSS. ${ }^{?}$

A primeira classificação utilizada foi a classificação FAB cujo principal fator prognóstico em termos de sobrevida global e risco de transformação leucêmica é a quantidade de mieloblastos medulares, que funciona quase como uma variável contínua. Já a classificação WHO leva em consideração não somente a contagem de mieloblastos medulares em um patamar máximo inferior (20\%) ao utilizado pela classificação FAB, mas também o número de linhagens hematopoiéticas com alterações displásicas na medula óssea e a citogenética no caso da síndrome do 5q-.

O principal e mais utilizado sistema prognóstico foi o desenvolvido por Greenberg, em 1997, que leva em consideração as seguintes variáveis: número de blastos medulares, grupo de risco citogenético e número de citopenias periféricas. Baseado nessas variáveis e na pontuação decorrente dessas, conseguem-se definir quatro grupos distintos quanto ao prognóstico (Tabelas 1 e 2). Já o sistema proposto por Dunkley, em 2002, alia as características dos grupos de risco

${ }^{1}$ Médico hematologista do Serviço de Hematologia do HC-1.

${ }^{2}$ Médica residente do Serviço de Hematologia do HC-1.

Correspondência: Alexandre Gustavo Apa

Instituto Nacional de Câncer- MS - Hospital do Câncer 1

Praça Cruz Vermelha, 23 - Centro

20230-130 - Rio de Janeiro-RJ 
Tabela 1

Fatores prognósticos de acordo com a classificação IPSS

\begin{tabular}{cccccc} 
Pontuação & 0 & 0,5 & 1,0 & 1,5 & 2,0 \\
\hline $\begin{array}{c}\text { Blastos na } \\
\text { M.O.(\%) }\end{array}$ & $<5$ & 5 a 10 & - & 11 a 20 & 21 a 30 \\
Cariótipo $^{1}$ & bom & intermediário & ruim & & \\
Citopenias $^{2}$ & 0 a 1 & 2 a 3 & & & \\
\hline
\end{tabular}

1. Cariótipo de baixo risco: normal, deleção do cromossomo $Y$, deleção do braço longo do cromossomo 5 ou deleção do braço longo do cromossomo 20. Cariótipo de risco intermediário: todas as demais alterações citogenéticas. Cariótipo de risco alto: alterações complexas (>3) ou alterações citogenéticas envolvendo o cromossomo 7.

2. Citopenias: neutrófilos $<1500 / \mathrm{mm}^{3}$ ou plaquetas $<100.000 / \mathrm{mm}^{3}$ ou hemoglobina $<10 \mathrm{~g} / \mathrm{dl}$.

Tabela 2

Grupos de risco de acordo com a soma de pontos na classificação IPSS

\begin{tabular}{lc}
\hline Grupo de Risco & Pontuação \\
\hline Baixo & 0 \\
Intermediário I & 0,5 a 1 \\
Intermediário II & 1,5 a 2 \\
Alto & $>2,5$ \\
\hline
\end{tabular}

Tabela 3

Fatores prognósticos de acordo com a classificação WHO presença de displasia e grupo de risco de acordo com classificação IPSS

\begin{tabular}{cccc}
\hline Sistema & Prognóstico & $\begin{array}{c}\text { Sobrevida média } \\
\text { (meses) }\end{array}$ & \\
\hline Linhagem & Displasia unilineal & 36.3 & $\mathrm{P}=0.01$ \\
(RA; RARS) & Displasia multilineal & 14.9 & \\
IPSS & Baixo & 39.1 & \\
& Intermediário 1 & 25.2 & \\
Linhagem + & Intermediário 2 & 8.3 & $\mathrm{P}<0.05$ \\
IPSS & Unilineal + baixo & 42.5 & \\
& Multilineal + baixo & 11.1 & \\
& Unilineal + & & \\
& intermediário - 1 & 29.6 & \\
& Multilineal + & 10.1 & \\
& intermediário- 1 & & \\
\hline
\end{tabular}

do sistema IPSS ao número de linhagens hematopoiéticas com alterações displásicas ${ }^{6}$ (Tabela 3).

Entretanto, o sistema prognóstico mais interessante e atual foi o proposto por Malcovati no ASH 2005 (WPSS), que alia os grupos de risco citogenéticos à presença ou não de dependência transfusional. ${ }^{7}$ Com isso conseguem-se definir cinco grupos de risco com diferenças estatiscamente significativas em termos de sobrevida global e de risco de transformação leucêmica: grupo de muito baixo risco (0 pontos), grupo de baixo risco (1 ponto), grupo de risco intermediário (2 pontos), grupo de alto risco (3 -4 pontos), grupo de muito alto risco (5-6 pontos) (Tabela 4).
Tabela 4

Fatores prognósticos de acordo com a classificação WPSS associação entre a classificação WHO, cariótipo e dependência transfusional

\begin{tabular}{ccccc}
\hline $\begin{array}{c}\text { Variável } \\
\text { prognóstica }\end{array}$ & Valor 0 & Valor 1 & Valor 2 & Valor 3 \\
\hline $\begin{array}{c}\text { Categoria } \\
\text { WHO }\end{array}$ & $\begin{array}{c}\text { RA, RARS, } \\
5 q-\end{array}$ & $\begin{array}{c}\text { RCMD e } \\
\text { RCMD- RS }\end{array}$ & RAEB-1 & RAEB- 2 \\
Cariótipo & Favorável & Intermediário & Desfavorável & \\
$\begin{array}{c}\text { Dependência } \\
\text { transfusional }\end{array}$ & Não & Sim & \\
\hline
\end{tabular}

É interessante observar que conforme o trabalho publicado por Malcovati, apenas o grupo de risco citogenético e a presença ou não de dependência transfusional se comportaram como variáveis independentes quando aplicadas à classificação WHO. ${ }^{1}$

Baseado nesses fatos, a proposta a ser formulada é a avaliação do sistema WPSS como um sistema prognóstico capaz de substituir o IPSS, a fim de melhor definir os grupos de risco e, conseqüentemente, as diferentes abordagens terapêuticas para cada um deles.

\section{Abstract}

The myelodysplastic syndromes represent a heterogeneous group of haematopoietic disorders characterized by ineffective haematopoiesis, peripheral cytopenias, hypercellular bone marrow and dysplastic haematopoiesis. Several laboratory and clinical features have been analysed as prognostic factors. The aim of this review is to evaluate the prognostic scoring systems focusing on overall survival and therapeutic approach. The WPSS evaluation includes both cytogenetic risk groups and transfusional necessities. It has five well-defined risk groups with statistical divergences related to overall survival and leukemic transformation risk. Our proposal is to evaluate the WPSS as a prognostic scoring system able to replace the IPSS, in order to establish a better definition of the risk groups and the different therapeutic approaches. Rev. bras. hematol. hemoter. 2006;28(3):198-200.

Key words: Prognostic; syndromes myelodysplastic.

\section{Referências Bibliográficas}

1. Malcovati L, Porta MG, Pascutto C, Invernizzi R, Boni M, Travagliano $\mathrm{E}$, et al. Prognostic factors and life expectancy in myelodysplastic syndromes classified according to WHO criteria: a basis for clinical decision making. Journal of Clinical Oncology 2005;23:7.5947.603 .

2. Morel P, Hebbar M, Lai JL, Duhamel A, Preudhomme C, Wattel E, et al. Cytogenetic analysis has strong independent prognostic value in de novo myelodysplastic syndromes and can be incorporated in a new scoring system: a report on 408 cases. Leukemia 1993;7: 1.315-1.323. 
3. Greenberg P, Cox C, LeBeau MM, et al. International scoring system for evolution prognosis in myelodysplastic syndromes. Blood 1987;89:2.079-2.088.

4. Bennett JM, Catovsky D, Daniel MT, et al. Proposals for the classification of the myelodysplastic syndromes. Br J Haematol 1982;51:189-199.

5. Vardiman JW, Harris NL, Brunning RD. The World Health Organization (WHO) classification of myeloid neoplasms. Blood 2002;100:2.292-2.302.

6. Dunkley SM, Manoharan A, Kwan YL. Myelodisplastic syndromes: prognostic significance of multilineage dysplasia in patients with refractory anemia or refractory anemia with ringed sideroblasts . Blood 2002;99:3.870-3.871.

7. Malcovati L, Germing U, Kuendgen A, Porta MGD, Invernizzi R , Giagounidis A, et al. A WHO classification - based prognostic scoring system (WPSS) for predicting survival in myelodysplastic syndromes. Blood 106; abstract 788.

Avaliação: Editor e dois revisores externos.

Conflito de interesse: não declarado

Recebido: 31/05/2006

Aceito após modificações: 11/09/2006 